\title{
Thionization Method of Glycosyl Urea and Carbamide Sugars
}

\section{Baktygul Ernazarova ${ }^{1}$, Aida Bakirova ${ }^{1}$, Asylkan Dzhumanazarova ${ }^{2}$, Zhypargul Abdullaeva ${ }^{3 *}$, Shamirbek Berkmamatov ${ }^{1}$, Gulsara Zhusupbaeva ${ }^{4}$}

\author{
${ }^{1}$ Department of Medical-Biological Disciplines, Zhalal-Abad State University, Zhalal-Abad, Kyrgyzstan \\ ${ }^{2}$ Institute of Chemistry and Phytotechnology, National Academy of Sciences, Bishkek, Kyrgyzstan \\ ${ }^{3}$ International Medical Faculty, Osh State University, Jolon Mamytov Campus, Osh, Kyrgyzstan \\ ${ }^{4}$ Zhalal-Abad Scientific Center, Southern Branch of the National Academy of Sciences, Zhalal-Abad, Kyrgyzstan \\ Email: ${ }^{*}$ jypar.science@oshsu.kg
}

How to cite this paper: Ernazarova, B. Bakirova, A., Dzhumanazarova, A., Abdullaeva, Z., Berkmamatov, Sh. and Zhusupbaeva, G. (2020) Thionization Method of Glycosyl Urea and Carbamide Sugars. International Journal of Organic Chemistry, 10, 111-122.

https://doi.org/10.4236/ijoc.2020.103008

Received: August 10, 2020

Accepted: September 14, 2020

Published: September 17, 2020

Copyright (c) 2020 by author(s) and Scientific Research Publishing Inc. This work is licensed under the Creative Commons Attribution International License (CC BY 4.0).

http://creativecommons.org/licenses/by/4.0/

\begin{abstract}
This work is describing a thionization method for glycosyl urea and carbamide of sugars by using the Lawesson's reagent. It is proposed the method based on the interaction of glycosyl urea and carbamide of sugars with the Lawessons reagent at a 1:1 ratio in the presence of a pyridine. As a result, sulfur-containing derivatives of sugar carbamides are obtained with the help of Lawesson's reagent. Obtained experimental data are indicating the developed new method for thionization of sugar carbamides, which opens up broad possibilities for synthesis of various carbohydrate derivatives of thiourea. Significance of this work is that, thiourea derivatives are promising bactericidal, fungicidal and anti-inflammatory drugs. Therefore, the preparation of thiourea derivatives and the study of their properties remain topical tasks in the field of chemistry.
\end{abstract}

\section{Keywords}

Glycosyl Urea, Glycosyl Thiourea Synthesis, Lawesson's Reagent, Anti-Inflammatory, Bactericidal, Fungicidal Properties

\section{Introduction}

Thiourea has great medicinal applications promising bactericidal [1], fungicidal [2] and anti-inflammatory drugs [3], as well as non-medicinal activities in the industry [4], analytical chemistry and metallurgy [5]. It is known that the replacement of the oxygen heteroatom by sulfur leads to a significant change in the spectrum of the biological activity of compounds [6]. Carbohydrate derivatives of thiourea can be obtained by the thionization of glycosyl ureas. In addi- 
tion, thionization of glycosyl urea derivatives is a practically unexplored area. The most common method for synthesis of glycosyl thiourea by isothiocyanate method was developed in 1914 by E. Fischer, which has several significant drawbacks, namely a multi-stage process, the use of expensive reagents (silver salt), the use of an expensive catalyst (platinum dioxide), aggressive and toxic reagents (sodium azide, bromine), deficient isothiocyanate, high pressure and reaction time [7]. We have developed a modified method for obtaining the Lawessons reagent, distinguished by its simplicity and accessibility [8], compared with the previous preparation method [9].

In this regard, it was of interest for us to develop a simplified and accessible method for obtaining of glycosyl thiourea derivatives. For these purposes, we have used the Lawessons reagent (2,4-bis(p-methoxyphenyl)-1,3-dithiadiphosphetan-2,4-disulfide) for thionization which allows us to eliminate the above-mentioned disadvantages of the isothiocyanate method of thionization as shown in Figure 1.

\section{Experimental}

A general method for thionization of sugar ureas conducted as following: 0.2 mmol of carbamide sugars, $0.2 \mathrm{mmol}$ of Lawessons reagent, and $3 \mathrm{ml}$ of abs. pyridine placed into the flask then boiled under reflux with the potassium chloride tube for 45 minutes. Then the solution evaporated under vacuum at the temperature of $50^{\circ} \mathrm{C}-60^{\circ} \mathrm{C}$ until dryness. $3 \mathrm{ml}$ of distilled water was added to this residue and refluxed for five minutes. Then the solution filtered and the filtrate evaporated. The residue recrystallized from alcohol: benzene mixture. Then precipitated crystals separated by filtration and dried in the air. Product yield was about $56 \%-60 \%$.

\section{Sample Characterizations}

Identification of the synthesized compounds carried out by using thin-layer chromatography on Silufol, IR-, NMR- ${ }^{13} \mathrm{C}, 1 \mathrm{H}$-spectroscopy, and elemental analysis. The ${ }^{13} \mathrm{C}$ NMR spectra were taken on a Bruker AM-300, $\mathrm{SF}=75.47 \mathrm{MHz}$ instruments with an operating frequency of $126 \mathrm{MG}$ at a temperature of $2950 \mathrm{~K}$, where TMS was used as an internal standard. The spectra taken in deuterated solvents-DMSO- $\mathrm{d}_{6}$. H1 NMR spectra were taken on a Bruker AM-300, SF = $300.13 \mathrm{MHz}$ instruments with an operating frequency of $500 \mathrm{MHz}$ at a temperature of $2930 \mathrm{~K}$, where TMS was used as an internal standard. The spectra taken

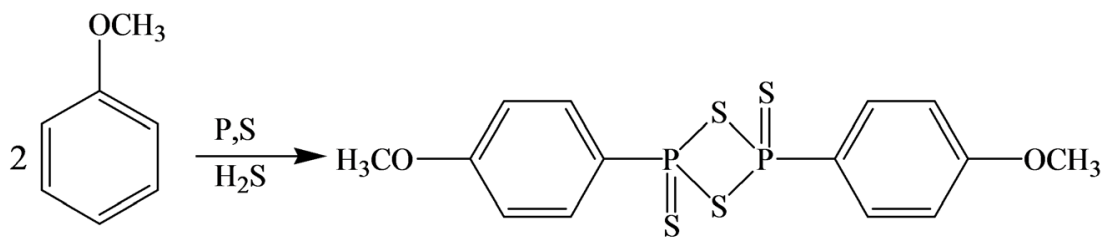

Figure 1. Simplified and accessible method for obtaining of glycosyl thiourea derivatives by the Lawessons reagent. 
in deuterated solvents-DMSO- $\mathrm{d}_{6}$. The IR spectra of the compounds obtained were obtained on spectrophotometers IKS-29, SpecordM-80 with the program "Soft Spectra", "SpectrumBXII" Fourier-IR spectrometer "NicoletAvatar 370" DTGS company Electron Corporation in the region of $500-4000 \mathrm{~cm}^{-1}$ (pressing with $\mathrm{KBr}$ ). The melting point of the synthesized compounds measured on a microheater Boetuis. The rate of temperature rise on the table was $4^{\circ} \mathrm{C}$ per minute. Elemental analysis of synthesized compounds was determined by colorimetric method Dyumo-Pregl and Schöniger on the device VM-20 (VLM-20g-M), SMD-1000 (VLM-1g). The control throughout the reaction and the purity of the synthesized compounds carried out using thin-layer chromatography (TLC) on SilufolUV-254 plates (sorbent: silica gel). System: chloroform:methanol (3:1), chloroform-ethanol-methyl ethyl ketone (1:2:1).

\section{Results and Discussions}

\subsection{Thionization of Glycosyl Urea}

We then applied the synthesized Lawessons reagent to the thionization of glycosylurea derivatives. The proposed method of thionization was based on the interaction of N-methyl-N1-( $\beta$-D-glycopyranosyl)-urea $(2-4)$ with the Lawessons (1) reagent at a 1:1 ratio of reagents in pyridine, where is formed $\mathrm{N}$-methyl- $\mathrm{N}^{1}$ ( $\beta$-D-glycopyranosyl)-thioureas (8 - 10) (Figure 2) [10]. This reaction proceeds quickly, so we could not fix the intermediate formation of the corresponding<smiles>[2H]NC(=O)NC[NH3+]</smiles>

$(2-4)$

(1)
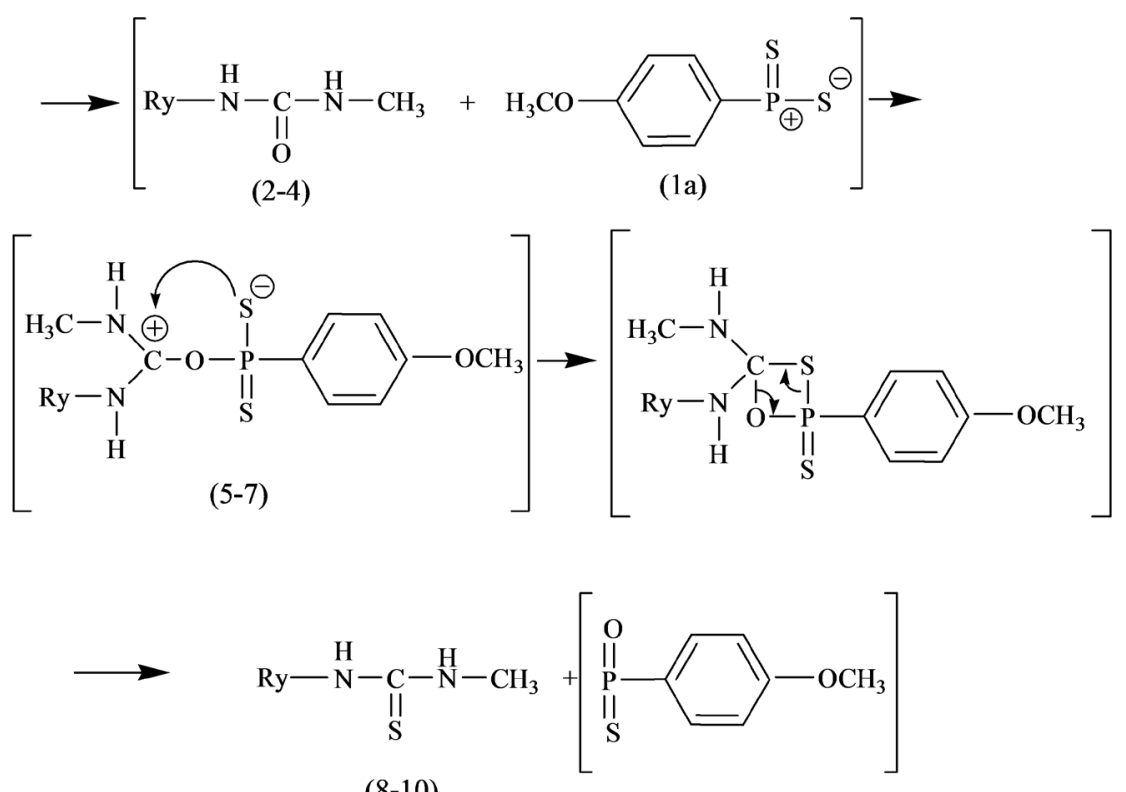

(8-10)

Figure 2. The thionization mechanism for glycosylurea (where Ry = xylose, glucose, and galactose). 
products. This is most likely due to the decomposition of the Lawessons (1) reagent into dithiometaphosphonate (1a), which has a resonant structure. The interaction of dithiometaphosphonate with glycosylmethyl urea (2-4) leads to the formation of intermediate cyclic thioketals $(5-7)$, with the subsequent decomposition, which produces the final products $(8-10)$.

The advantage of this method is the simplicity, acceleration of the process, and elimination from the use of high-pressure technological processes and expensive platinum dioxide, poisonous sodium azide, and bromine reagents. Compounds synthesized by the new method are crystalline substances possessing chemical stability. They can be stored without decomposition at room temperature, stable under thin-layer chromatography (TLC) conditions. The individuality and structure of the target products were confirmed by the methods of nuclear magnetic resonance ${ }^{13} \mathrm{C}$ (NMR), ${ }^{1} \mathrm{H}$ NMR, infrared (IR)-spectroscopy, also elemental analysis. In the ${ }^{13} \mathrm{C}$ NMR and ${ }^{1} \mathrm{H}$ NMR spectra of as-synthesized compounds, we observed nuclei signals belonging to all presenting compounds (Table 1).

In the IR spectra, in particular of N-methyl-N1-( $\beta$-D-glucopyranosyl)-thiourea, a broadband was observed in the region of $3000-3550 \mathrm{~cm}^{-1}$, which is characteristic of the stretching vibrations of the $\mathrm{OH}$ - and $\mathrm{NH}$ - groups. Absorption bands in the regions of 1024 and $1108 \mathrm{~cm}^{-1}$ are related to the stretching vibrations of the carbohydrate ring. The presence of signals at $926 \mathrm{~cm}^{-1}$ is indicating $\beta$-position of the pyranotic ring. Oscillations in the region of $1256-1441 \mathrm{~cm}^{-1}$ can be attributed to the valence vibrations of the group $(\mathrm{C}=\mathrm{S})$. Peaks in the region of $2836 \mathrm{~cm}^{-1}$ are attributing to $\mathrm{CH}_{3}$ group vibrations.

Important structural information was obtained by the $1 \mathrm{H}$ NMR spectra. According to the proton magnetic resonance (PMR) spectrum, the structures of the products obtained are compounds formed from the glycosylamine bond with the $\beta$-arrangement of glucosyl methylthiourea. The low-field part of the PMR spectrum for the $\mathrm{N}$-methyl- $\mathrm{N}^{1}$-( $\beta$-D-glucopyranosyl)-thiourea contains signals that appearing as a multiplet centered at $3.6 \mathrm{ppm}-3.8 \mathrm{ppm}$, respectively, belonging to the fifth axial and the fifth equatorial hydrogen atom of the carbon-water ring. The equatorial hydrogen atom at C-2 appears as a doublet with a chemical shift at $3.8 \mathrm{ppm}$. A signal in the form of a broadened singlet, belonging to the protons of the methyl group $\mathrm{CH}_{3}$, is observed in the region of $2.7 \mathrm{ppm}$. The signal in the region of $4.6 \mathrm{ppm}-4.85 \mathrm{ppm}$ refers to carbohydrate rings.

${ }^{13} \mathrm{C}$ NMR spectra of $\mathrm{N}$-methyl- ${ }^{1}$-( $\beta$-D-glucopyranosyl) thiourea, the anomeric carbon atoms were found in the range of $\delta 60.69-81.09 \mathrm{ppm}$, which also testifies favor for the $\beta$-configuration of the glyosidic bond. Signals in the region of $60.69 \mathrm{ppm}$ and $\delta 69.42$ suggest that the glucoside residue in the compounds under discussion is in the pyranose form. Signals in the region of $\delta 26.28 \mathrm{ppm}$ belonging to the methyl group, $\mathrm{C}=\mathrm{S}$ signals were observed in the field of $\approx 160.13$ ppm. The set of spectral characteristics of the synthesized compounds do not doubt that all the substances obtained are individual compounds. 


\subsection{Synthesis of 2,4-Bis(p-methoxyphenyl)-1,3- dithiadiphosphetan-2,4-disulfide}

In a flask equipped with a reflux condenser and a calcium chloride tube, $3.661 \mathrm{~g}$ $(0.114 \mathrm{~mol})$ of sulfur and $1.4 \mathrm{~g}(0.045 \mathrm{~mol})$ of red phosphorus placed and boiled. The reaction mass was then cooled, $12 \mathrm{ml}(0.11 \mathrm{~mol})$ of anisole was added and boiled. After cooling up to the room temperature, the precipitated crystals filtered and washed with absolute ether and benzene, and then recrystallized from absolute toluene. Product yield: $4.36 \mathrm{~g},(47.8 \%), \mathrm{T}_{\text {melt }}=228^{\circ} \mathrm{C}-229^{\circ} \mathrm{C}$. IR spectrum $\left(\mathrm{KBr}, \mathrm{v}, \mathrm{cm}^{-1}\right): 689(\mathrm{P}=\mathrm{S}), 615(\mathrm{P}=\mathrm{C}), 1022,1095,1180$ (R-O-CH3), 1267, 1294, 1308, 1458, 1493, 1592 (arom.). $\mathrm{C}_{14} \mathrm{H}_{14} \mathrm{O}_{2} \mathrm{P}_{2} \mathrm{~S}_{4} 404.475$ Calculated \%: C-41.57; H-3.49; P-15.32; S-31.71. Found \%: C-41.95; H-3.78; R-15.1; S-32.0.

\subsection{Thionization of Carbamide Sugars}

Lawessons reagent was used by us for thionization of carbamide derivatives of sugars to obtain thiocarbamide derivatives, 4 -(N- $\beta$-D-glycopyranosyl)-thiosemicarbazide [11], previously synthesized by us, $\mathrm{N}$ - $(\beta$-D-glycopyranosyl) phenylsemicarbazide, 2,3,4,6-tetra-O-acetyl-( $\beta$-D-glycopyranosyl)-carbamoyl diethylenediamine, 1-[(N-glycopyranosyl) carbamoyl]-3,5-dimethylpyrazole [10] [12] [13] [14] as shown in Figure 3.

The reaction proceeds smoothly with the interaction of carbamide derivatives of sugars with Lawesson reagent in pyridine, for 20 minutes and leads to the formation of the corresponding 4-(N- $\beta$-D-glycopyranosyl)-thiosemicarbazide, $\mathrm{N}$-( $\beta$-D-glycopyranosyl) phenylthiosemicarbazides, 1 -[(N- $\beta$-glycopyranosyl) thiocarbamoyl]-3,5-dimethylpyrazoles, 2,3,4,6-tetra-O-acetyl-( $\beta$-D-glycopyranosyl)thiocarbamoyl diethylenediamine diamine (Figure 4). The course of reactions and compositions of obtained compounds was controlled and carried out using the methods of thin-layer and paper chromatography in the systems: chloroform-ethanol-methyl ethyl ketone (1:2:1).

Compounds synthesized by the method are the crystalline substances possessing chemical stability. They can be stored without decomposition at room temperature, stable under TLC.

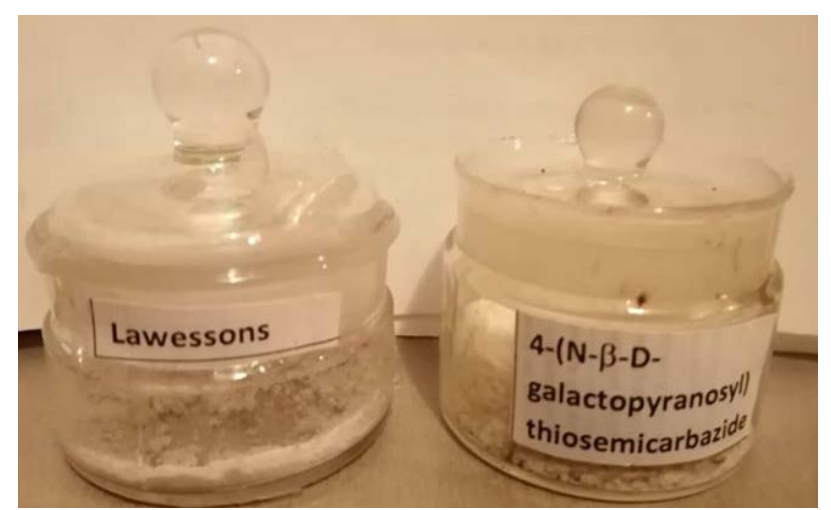

Figure 3. Synthesized samples of the Lawessons reagent and 4 -(N- $\beta$-D-glycopyranosyl)-thiosemicarbazide. 


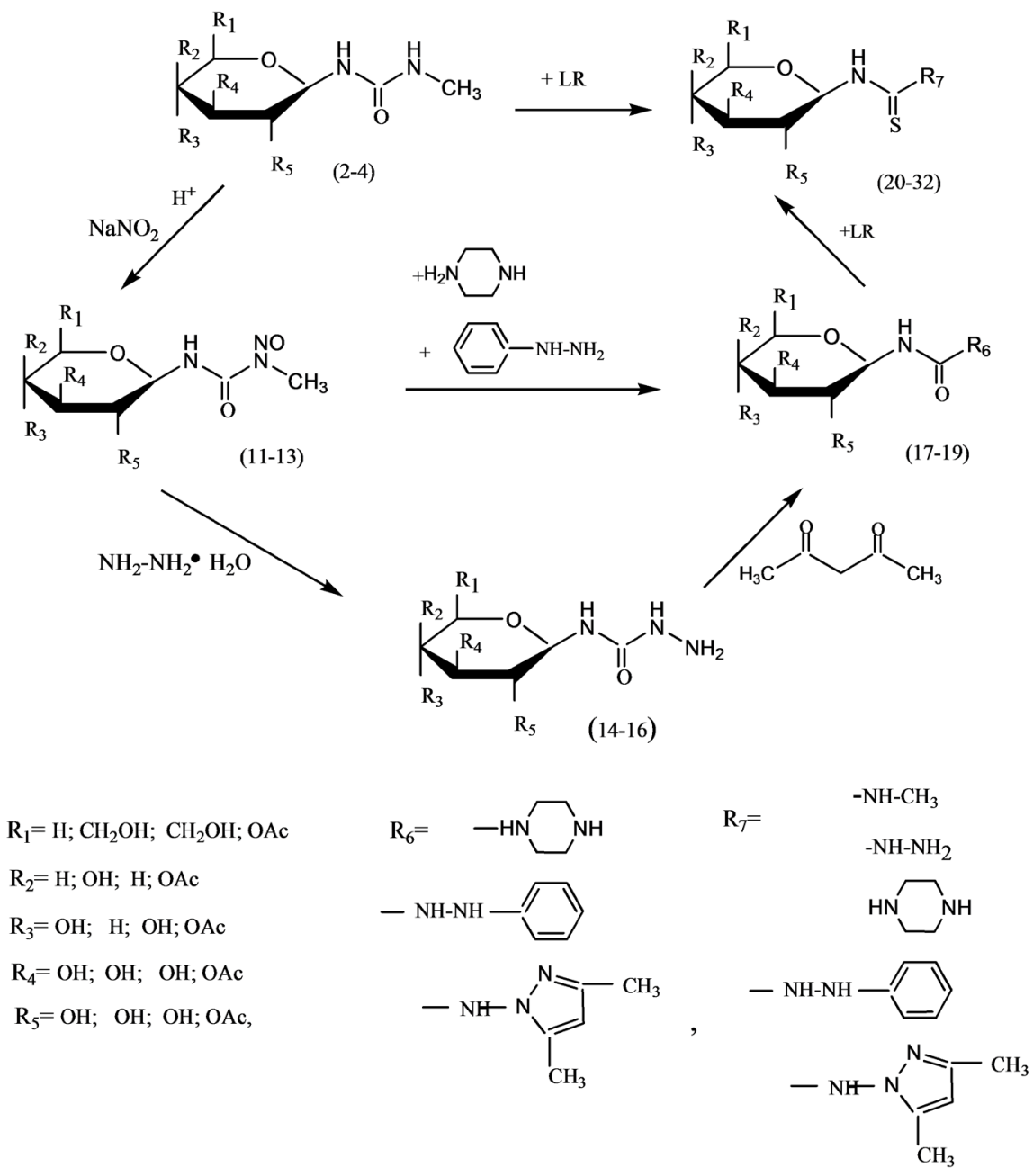

Figure 4. Thionization reaction for carbamide derivatives of sugars.

Individuality and structures of the target products confirmed by physicochemical methods of ${ }^{13} \mathrm{C}$ and $\mathrm{H}^{1}$ NMR, IR spectroscopy analyses. Results were presented in Tables 1-4, characteristic maximums of sugar thiocarbamides in the IR spectrum absorption bands, and chemical shifts for ${ }^{13} \mathrm{C}$ NMR of sugar thiocarbamides in Figure 5, Figure 6.

\section{Conclusion}

Thionization method of glycosyl urea and carbamide compounds of sugars is presented in this article. Obtained compounds are crystalline substances possessing chemical stability, and open up new ways of synthesizing various carbohydrate derivatives of thiourea. For thionization of carbamide derivatives of sugars, the Lawessons reagent is used to obtain thiocarbamide derivatives, 4 -(N- $\beta$-D-glycopyranosyl)-semicarbazide which allows eliminating disadvantages of the isothiocyanate use. Obtained experimental data is useful for thionization of sugar derivatives and provides possibilities for the synthesis of various carbohydrate derivatives of thiourea. 
Table 1. Physio-chemical property of thiocarbamide sugars.

\begin{tabular}{|c|c|c|c|c|c|c|c|c|c|}
\hline \multirow{2}{*}{ No. } & \multirow{2}{*}{ Compounds } & \multirow{2}{*}{ Formula } & \multirow{2}{*}{$\mathrm{T}_{\text {melt }}{ }^{\circ} \mathrm{C}$} & \multirow{2}{*}{$\begin{array}{c}\text { Output } \\
\%\end{array}$} & \multirow{2}{*}{$\mathrm{Rf}$} & \multicolumn{4}{|c|}{$\begin{array}{l}\text { Calculated \% (found \%) } \\
\text { elemental compositions }\end{array}$} \\
\hline & & & & & & $\mathrm{C}$ & $\mathrm{H}$ & $\mathbf{N}$ & $S$ \\
\hline 20 & $\mathrm{~N}$-methyl-N $\mathrm{N}^{1}-(\beta$-D-xylopyranosyl)-thiourea & $\mathrm{C}_{7} \mathrm{H}_{14} \mathrm{~N}_{2} \mathrm{O}_{4} \mathrm{~S}$ & $122-125$ & 53 & 0.2 & $\begin{array}{c}37.82 \\
(37.97)\end{array}$ & $\begin{array}{c}6.34 \\
(6.45)\end{array}$ & $\begin{array}{c}12.60 \\
(12.80)\end{array}$ & $\begin{array}{c}14.42 \\
(14.60)\end{array}$ \\
\hline 21 & $\mathrm{~N}$-methyl- $\mathrm{N}^{1}-(\beta$-D-galactopyranosyl)-thiourea & $\mathrm{C}_{8} \mathrm{H}_{16} \mathrm{~N}_{2} \mathrm{O}_{5} \mathrm{~S}$ & $168-170$ & 48 & 0.8 & $\begin{array}{c}38.08 \\
(38.25)\end{array}$ & $\begin{array}{c}6.39 \\
(6.50)\end{array}$ & $\begin{array}{c}11.10 \\
(11.29)\end{array}$ & $\begin{array}{c}12.71 \\
(12.85)\end{array}$ \\
\hline 22 & $\mathrm{~N}$-methyl- $\mathrm{N}^{1}$-( $\beta$-D-glucopyranosyl)-thiourea & $\mathrm{C}_{8} \mathrm{H}_{16} \mathrm{~N}_{2} \mathrm{O}_{5} \mathrm{~S}$ & $165-167$ & 58 & 0.6 & $\begin{array}{c}38.08 \\
(38.24)\end{array}$ & $\begin{array}{c}6.39 \\
(6.50)\end{array}$ & $\begin{array}{c}11.10 \\
(11.25)\end{array}$ & $\begin{array}{c}12.71 \\
(12.87)\end{array}$ \\
\hline 23 & $\begin{array}{l}\mathrm{N} \text { - }(\beta \text {-D-xylopyranosyl }) \\
\text { phenylthiosemicarbazide }\end{array}$ & $\mathrm{C}_{12} \mathrm{H}_{17} \mathrm{~N}_{3} \mathrm{O}_{4} \mathrm{~S}$ & $149-152$ & 52 & 0.87 & $\begin{array}{c}48.14 \\
(48.04)\end{array}$ & $\begin{array}{c}5.72 \\
(5.98)\end{array}$ & $\begin{array}{c}14.03 \\
(13.94)\end{array}$ & $\begin{array}{c}10.71 \\
(10.86)\end{array}$ \\
\hline 24 & $\begin{array}{l}\mathrm{N}-(\beta \text {-Dgalactopyranosyl }) \\
\text { phenylthiosemicarbazide }\end{array}$ & $\mathrm{C}_{13} \mathrm{H}_{19} \mathrm{~N}_{3} \mathrm{O}_{5} \mathrm{~S}$ & $137-140$ & 56 & 0.84 & $\begin{array}{c}47.40 \\
(47.21)\end{array}$ & $\begin{array}{c}5.81 \\
(5.98)\end{array}$ & $\begin{array}{c}12.75 \\
(12.94)\end{array}$ & $\begin{array}{c}9.73 \\
(9.45)\end{array}$ \\
\hline 25 & $\begin{array}{l}\mathrm{N} \text {-( } \beta \text {-D-glucopyranosyl }) \\
\text {-phenylthiosemicarbazide }\end{array}$ & $\mathrm{C}_{13} \mathrm{H}_{19} \mathrm{~N}_{3} \mathrm{O}_{5} \mathrm{~S}$ & $142-144$ & 55 & 0.75 & $\begin{array}{c}47.40 \\
(47.23)\end{array}$ & $\begin{array}{c}5.81 \\
(6.05)\end{array}$ & $\begin{array}{c}12.75 \\
(12.92)\end{array}$ & $\begin{array}{c}9.73 \\
(9.94)\end{array}$ \\
\hline 26 & $\begin{array}{c}1-[(\mathrm{N}-\beta \text {-xylopyranosyl)-thiocarbamoyl }] \\
\text {-3,5-dimethylpyrazole }\end{array}$ & $\mathrm{C}_{11} \mathrm{H}_{17} \mathrm{~N}_{3} \mathrm{O}_{4} \mathrm{~S}$ & $116-117$ & 58 & 0.89 & $\begin{array}{c}45.98 \\
(46.07)\end{array}$ & $\begin{array}{c}5.96 \\
(6.13)\end{array}$ & $\begin{array}{c}14.62 \\
(14.80)\end{array}$ & $\begin{array}{r}11.15 \\
(11.25)\end{array}$ \\
\hline 27 & $\begin{array}{c}1-[(\mathrm{N}-\beta \text {-galactopyranosyl)-thiocarbamoyl }] \\
-3,5 \text {-dimethylpyrazole }\end{array}$ & $\mathrm{C}_{12} \mathrm{H}_{19} \mathrm{~N}_{3} \mathrm{O}_{5} \mathrm{~S}$ & $146-148$ & 61 & 0.6 & $\begin{array}{c}45.41 \\
(45.67)\end{array}$ & $\begin{array}{c}6.03 \\
(6.15)\end{array}$ & $\begin{array}{c}13.24 \\
(13.40)\end{array}$ & $\begin{array}{c}10.10 \\
(10.25)\end{array}$ \\
\hline 28 & $\begin{array}{c}1-[(\mathrm{N}-\beta \text {-glucopyranosyl)-thiocarbamoyl }] \\
\text {-3,5-dimethylpyrazole }\end{array}$ & $\mathrm{C}_{12} \mathrm{H}_{19} \mathrm{~N}_{3} \mathrm{O}_{5} \mathrm{~S}$ & $150-151$ & 56.5 & 0.7 & $\begin{array}{c}45.41 \\
(45.52)\end{array}$ & $\begin{array}{c}6.03 \\
(5.96)\end{array}$ & $\begin{array}{c}13.24 \\
(13.45)\end{array}$ & $\begin{array}{c}10.10 \\
(10.35)\end{array}$ \\
\hline 29 & 4-(N- $\beta$-D-xylopyranosyl)thiosemicarbazide & $\mathrm{C}_{6} \mathrm{H}_{13} \mathrm{~N}_{3} \mathrm{O}_{4} \mathrm{~S}$ & $217-219$ & 35.6 & 0.8 & $\begin{array}{c}32.28 \\
(32.40)\end{array}$ & $\begin{array}{c}5.86 \\
(6.10)\end{array}$ & $\begin{array}{c}18.82 \\
(18.97)\end{array}$ & $\begin{array}{c}14.36 \\
(14.47)\end{array}$ \\
\hline 30 & 4-(N- $\beta$-D-galactopyranosyl)thiosemicarbazide & $\mathrm{C}_{7} \mathrm{H}_{15} \mathrm{~N}_{3} \mathrm{O}_{5} \mathrm{~S}$ & $210-211$ & 46 & 0.9 & $\begin{array}{c}33.19 \\
(33.36)\end{array}$ & $\begin{array}{c}5.96 \\
(6.28)\end{array}$ & $\begin{array}{c}16.59 \\
(16.75)\end{array}$ & $\begin{array}{c}12.65 \\
(12.98)\end{array}$ \\
\hline 31 & 4-(N- $\beta$-D-glucopyranosyl)thiosemicarbazide & $\mathrm{C}_{7} \mathrm{H}_{15} \mathrm{~N}_{3} \mathrm{O}_{5} \mathrm{~S}$ & $205-206$ & 37 & 0.6 & $\begin{array}{c}33.19 \\
(33.32)\end{array}$ & $\begin{array}{c}5.96 \\
(6.26)\end{array}$ & $\begin{array}{c}16.59 \\
(16.64)\end{array}$ & $\begin{array}{c}12.65 \\
(12.85)\end{array}$ \\
\hline 32 & $\begin{array}{l}\text { 2,3,4,6-tetra-O-acetyl-( } \beta \text {-D-glycopyranosyl) } \\
\text {-thiocarbamoyl diethylene diamine) }\end{array}$ & $\mathrm{C}_{22} \mathrm{H}_{35} \mathrm{~N}_{3} \mathrm{O}_{9} \mathrm{~S}$ & $135-137$ & 62.5 & 0.84 & $\begin{array}{c}51.05 \\
(50.97)\end{array}$ & $\begin{array}{c}6.81 \\
(6.98)\end{array}$ & $\begin{array}{c}8.11 \\
(8.33)\end{array}$ & $\begin{array}{c}6.19 \\
(6.35)\end{array}$ \\
\hline
\end{tabular}


Table 2. Characteristic maximums of sugar thiocarbamides in the IR spectrum absorption bands.

\begin{tabular}{|c|c|c|c|c|c|c|}
\hline \multirow{2}{*}{ No. } & \multicolumn{3}{|c|}{$\begin{array}{l}\text { Fluctuations in the carbohydrate } \\
\text { fragment, } \mathrm{v}, \mathrm{cm}^{-1}\end{array}$} & \multicolumn{3}{|c|}{$\begin{array}{l}\text { Aglycone fluctuations, } \\
\qquad \mathrm{v}, \mathrm{cm}^{-1}\end{array}$} \\
\hline & $-\mathrm{C}-\mathrm{O}-$ & $\mathrm{OH}$ & $\beta$-form & $\mathrm{N}-\mathrm{H}$ & $\mathrm{C}=\mathrm{S}$ & Other signals \\
\hline 20 & $\begin{array}{l}1029 \\
1145\end{array}$ & $\begin{array}{l}2838 \\
3046\end{array}$ & 955 & $\begin{array}{l}3288 \\
1658 \\
1602\end{array}$ & $\begin{array}{l}1257 \\
1403 \\
1439\end{array}$ & $\begin{array}{l}2958 \\
\left(\mathrm{CH}_{3}\right)\end{array}$ \\
\hline 21 & $\begin{array}{l}1024 \\
1108\end{array}$ & 2939 & 926 & $\begin{array}{l}3403 \\
1536 \\
1649\end{array}$ & $\begin{array}{l}1256 \\
1461 \\
1441\end{array}$ & $\begin{array}{c}2836 \\
\left(\mathrm{CH}_{3}\right)\end{array}$ \\
\hline 22 & $\begin{array}{l}1030 \\
1074 \\
1144\end{array}$ & $\begin{array}{l}2958 \\
3046\end{array}$ & 949 & $\begin{array}{l}3288 \\
1572 \\
1602\end{array}$ & $\begin{array}{l}1257 \\
1453\end{array}$ & $\begin{array}{c}2837 \\
\left(\mathrm{CH}_{3}\right)\end{array}$ \\
\hline 23 & 1233 & 3463 & 912 & $\begin{array}{l}3269 \\
1525 \\
1748\end{array}$ & $\begin{array}{l}1364 \\
1448\end{array}$ & $\begin{array}{c}482-834 \\
(\mathrm{C}-\mathrm{H} \text { arom })\end{array}$ \\
\hline 24 & 1228 & 3339 & 915 & $\begin{array}{l}2960 \\
1536 \\
1750\end{array}$ & $\begin{array}{l}1371 \\
1499\end{array}$ & $\begin{array}{c}492-831 \\
(\mathrm{C}-\mathrm{H} \text { arom })\end{array}$ \\
\hline 25 & 1230 & 3343 & 910 & $\begin{array}{l}2950 \\
1532 \\
1730\end{array}$ & $\begin{array}{l}1360 \\
1475\end{array}$ & $\begin{array}{c}475-833 \\
(\mathrm{C}-\mathrm{H} \text { arom })\end{array}$ \\
\hline 26 & 1216 & 3453 & 909 & $\begin{array}{l}3269 \\
1533 \\
1626\end{array}$ & 1424 & $\begin{array}{c}2919 \\
\left(\mathrm{CH}_{3}\right)\end{array}$ \\
\hline 27 & 1220 & 3443 & 902 & $\begin{array}{l}3250 \\
1536 \\
1726\end{array}$ & 1440 & $\begin{array}{c}2930 \\
\left(\mathrm{CH}_{3}\right)\end{array}$ \\
\hline 28 & 1225 & 3450 & 905 & $\begin{array}{l}3260 \\
1538 \\
1722\end{array}$ & 1445 & $\begin{array}{l}2925 \\
\left(\mathrm{CH}_{3}\right)\end{array}$ \\
\hline 29 & 1108 & $\begin{array}{l}3000 \\
3500\end{array}$ & 901 & $\begin{array}{l}3251 \\
1505\end{array}$ & 1461 & $\begin{array}{c}3377 \\
\left(\mathrm{NH}_{2}\right)\end{array}$ \\
\hline 30 & 1133 & $\begin{array}{l}3000 \\
3400\end{array}$ & 908 & $\begin{array}{l}3233 \\
1530\end{array}$ & 1332 & $\begin{array}{c}3369 \\
\left(\mathrm{NH}_{2}\right)\end{array}$ \\
\hline 31 & 1140 & $\begin{array}{l}3000 \\
3400\end{array}$ & 905 & $\begin{array}{l}3235 \\
1520\end{array}$ & 1350 & $\begin{array}{c}3360 \\
\left(\mathrm{NH}_{2}\right)\end{array}$ \\
\hline 32 & 1228 & - & 918 & $\begin{array}{l}3371 \\
1541\end{array}$ & $\begin{array}{l}1367 \\
1421\end{array}$ & $\begin{array}{c}601(\mathrm{CH} \text { arom }) \\
1332(\mathrm{OAc})\end{array}$ \\
\hline
\end{tabular}


Table 3. Chemical shifts of thiocarbamide sugar protons.

\begin{tabular}{|c|c|c|c|c|}
\hline \multirow{2}{*}{ No. } & \multicolumn{2}{|c|}{ Carbonaceous part } & \multicolumn{2}{|c|}{ Aglycone part } \\
\hline & $\mathrm{CH}$ & $\mathrm{OH}$ & $\mathrm{NH}$ & Other signals \\
\hline 20 & $\begin{array}{l}4.7 \text { broad singlet } \\
3.2-3.7 \text { multiplet }\end{array}$ & $\begin{array}{c}4.5-4.8 \text { broad singlet } \\
(3 \mathrm{OH})\end{array}$ & 5.4 singlet $(1 \mathrm{H})$ & $\begin{array}{l}2.7 \text { broad singlet } \\
\qquad(3 \mathrm{H})\left(\mathrm{CH}_{3}\right)\end{array}$ \\
\hline 21 & $\begin{array}{c}3.5 \text { triplet } \\
3.6-3.8 \text { multiplet }\end{array}$ & $\begin{array}{c}4.6-4.8 \text { broad singlet } \\
(4 \mathrm{OH})\end{array}$ & 5.4 singlet $(1 \mathrm{H})$ & $\begin{array}{l}2.7 \text { broad singlet } \\
\qquad(3 \mathrm{H})\left(\mathrm{CH}_{3}\right)\end{array}$ \\
\hline 22 & $3.2-3.9$ multiplet & $\begin{array}{c}4.6-4.7 \text { broad singlet } \\
(4 \mathrm{OH})\end{array}$ & 5.4 singlet $(1 \mathrm{H})$ & $\begin{array}{l}2.7 \text { broad singlet } \\
\qquad(3 \mathrm{H})\left(\mathrm{CH}_{3}\right)\end{array}$ \\
\hline 23 & 3.2 - 4 multiplet & $\begin{array}{l}4.8 \text { broad singlet } \\
\qquad(3 \mathrm{OH})\end{array}$ & $\begin{array}{l}7.2 \text { duplet }(1 \mathrm{H}) \\
6.8 \text { duplet }(1 \mathrm{H})\end{array}$ & $\begin{array}{c}2.0-2.2 \\
(\operatorname{arom} 5 \mathrm{H})\end{array}$ \\
\hline 24 & $\begin{array}{l}4.2 \text { broad singlet } \\
3.5 \text { triplet } \\
3.6-4.0 \text { multiplet }\end{array}$ & $\begin{array}{c}4.6-4.7 \text { broad singlet } \\
(4 \mathrm{OH})\end{array}$ & $\begin{array}{l}7.7 \text { duplet }(1 \mathrm{H}) \\
7.7 \text { duplet }(1 \mathrm{H})\end{array}$ & $\begin{array}{l}2.0-2.4 \text { multiplet } \\
\quad(\operatorname{arom} 5 \mathrm{H})\end{array}$ \\
\hline 25 & $\begin{array}{c}4.0 \text { singlet } \\
3.4 \text { triplet } \\
3.49-3.46 \text { multiplet }\end{array}$ & $\begin{array}{l}4.8 \text { singlet } \\
(4 \mathrm{OH})\end{array}$ & $\begin{array}{l}7.5 \text { duplet }(1 \mathrm{H}) \\
7.6 \text { duplet }(1 \mathrm{H})\end{array}$ & $\begin{array}{c}2.0-2.2 \\
(\operatorname{arom} 5 \mathrm{H})\end{array}$ \\
\hline 26 & $\begin{array}{c}3.7 \text { singlet } \\
3.2 \text { triplet } \\
3.5-3.7 \text { multiplet }\end{array}$ & $\begin{array}{l}4.5 \text { singlet } \\
(3 \mathrm{OH})\end{array}$ & 6.0 duplet $(1 \mathrm{H})$ & $\begin{array}{l}2.5 \text { broad singlet } \\
(6 \mathrm{H})\left(\mathrm{CH}_{3}\right) \\
5.8 \text { singlet } \\
\text { (pyrazole } \mathrm{CH} 1 \mathrm{H})\end{array}$ \\
\hline 27 & $\begin{array}{c}3.79 \text { singlet } \\
3.4 \text { triplet } \\
3.5-3.76 \text { multiplet }\end{array}$ & $\begin{array}{l}4.7 \text { singlet } \\
(4 \mathrm{OH})\end{array}$ & 6.2 duplet $(1 \mathrm{H})$ & $\begin{array}{l}2.8 \text { broad singlet } \\
(6 \mathrm{H})\left(\mathrm{CH}_{3}\right) \\
5.9 \text { singlet } \\
\text { (pyrazole } \mathrm{CH} 1 \mathrm{H})\end{array}$ \\
\hline 28 & $\begin{array}{c}3.79 \text { singlet } \\
3.4 \text { triplet } \\
3.49-3.76 \text { multiplet }\end{array}$ & $\begin{array}{l}4.82 \text { singlet } \\
\quad(4 \mathrm{OH})\end{array}$ & 6.2 duplet $(1 \mathrm{H})$ & $\begin{array}{l}2.8 \text { broad singlet } \\
(6 \mathrm{H})\left(\mathrm{CH}_{3}\right) \\
5.9 \text { singlet } \\
\text { (pyrazole } \mathrm{CH} 1 \mathrm{H})\end{array}$ \\
\hline 29 & $\begin{array}{c}3.88 \text { quartet } \\
3.41 \text { triplet } \\
3.49-3.76 \text { multiplet }\end{array}$ & $\begin{array}{l}2.0 \text { broad singlet } \\
(3 \mathrm{OH})\end{array}$ & 4.81 duplet $(1 \mathrm{H})$ & $\begin{array}{l}2 \text { broad singlet } \\
\left(\mathrm{NH}_{2} ; \mathrm{NH}\right)(3 \mathrm{H})\end{array}$ \\
\hline 30 & $\begin{array}{c}3.79 \text { multiplet } \\
3.4 \text { triplet } \\
3.48-3.76 \text { multiplet }\end{array}$ & $\begin{array}{l}2.1 \text { broad singlet } \\
(4 \mathrm{OH})\end{array}$ & 4.83 duplet $(1 \mathrm{H})$ & $\begin{array}{l}2 \text { broad singlet } \\
\left(\mathrm{NH}_{2} ; \mathrm{NH}\right)(3 \mathrm{H})\end{array}$ \\
\hline 31 & $\begin{array}{c}3.76 \text { multiplet } \\
3.4 \text { triplet } \\
3.48-3.76 \text { multiplet }\end{array}$ & $\begin{array}{l}2.1 \text { broad singlet } \\
(4 \mathrm{OH})\end{array}$ & 4.80 duplet $(1 \mathrm{H})$ & $\begin{array}{l}2 \text { broad singlet } \\
\left(\mathrm{NH}_{2} ; \mathrm{NH}\right)(3 \mathrm{H})\end{array}$ \\
\hline 32 & $\begin{array}{l}4.16 \text { multiplet } \\
3.6 \text { - } 3.91 \text { multiplet }\end{array}$ & $\begin{array}{c}4.09-4.34 \text { multiplet } \\
\left(-\mathrm{CH}_{2} \mathrm{O}\right)(8 \mathrm{H}) \\
2.0 \text { broad singlet } \\
\left(\mathrm{CH}_{3} 12 \mathrm{H}\right)\end{array}$ & 4.72 duplet $(1 \mathrm{H})$ & $\begin{array}{c}3.37,2.67,3.57,2.67,2.0 \\
\left(\mathrm{CH}_{2} ; \mathrm{NH} \text { amine }\right)\end{array}$ \\
\hline
\end{tabular}




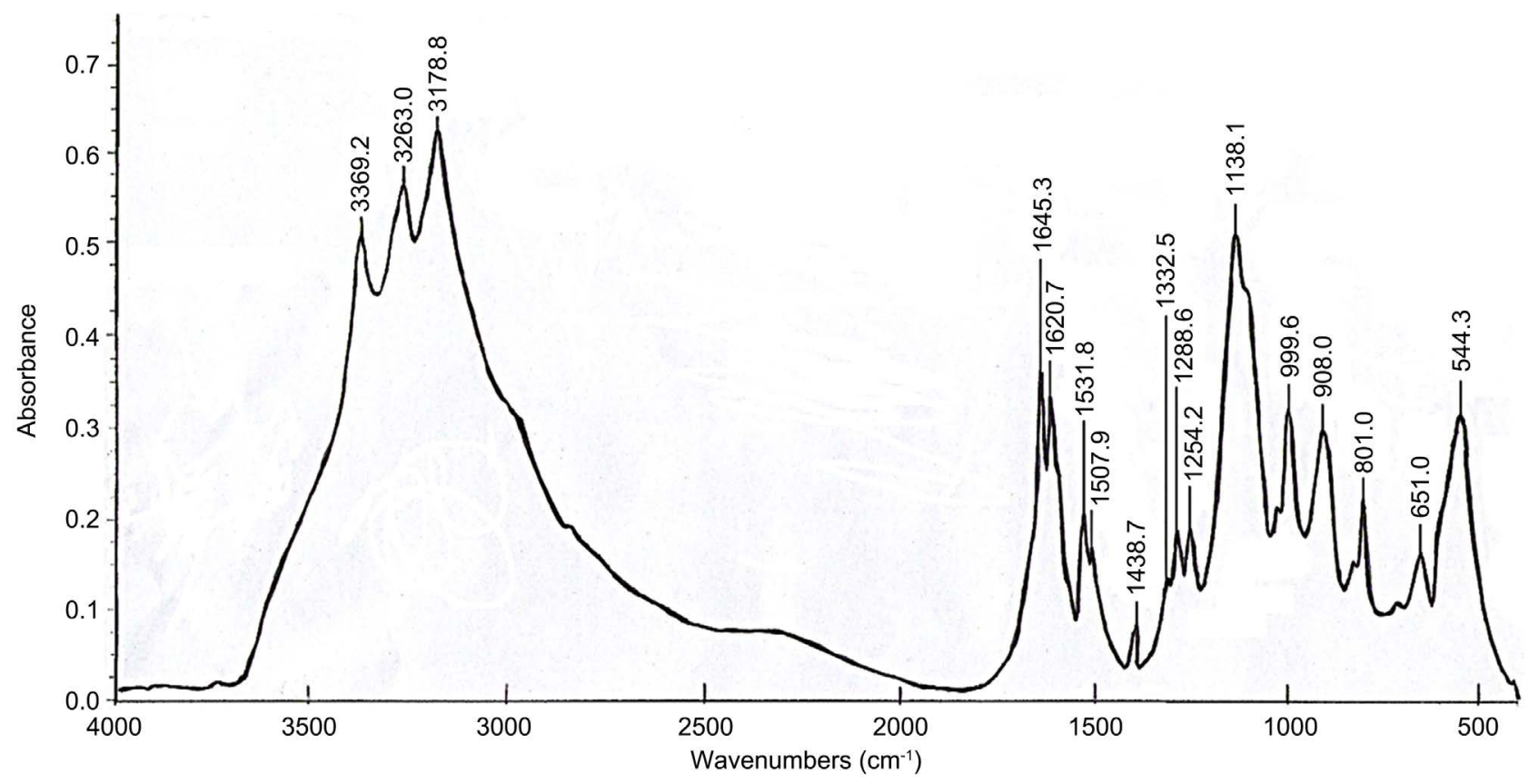

Figure 5. Characteristic maximums of sugar thiocarbamide 4-(N- $\beta$-D-galactopyranosyl) thiosemicarbazide in the IR spectrum absorption bands.

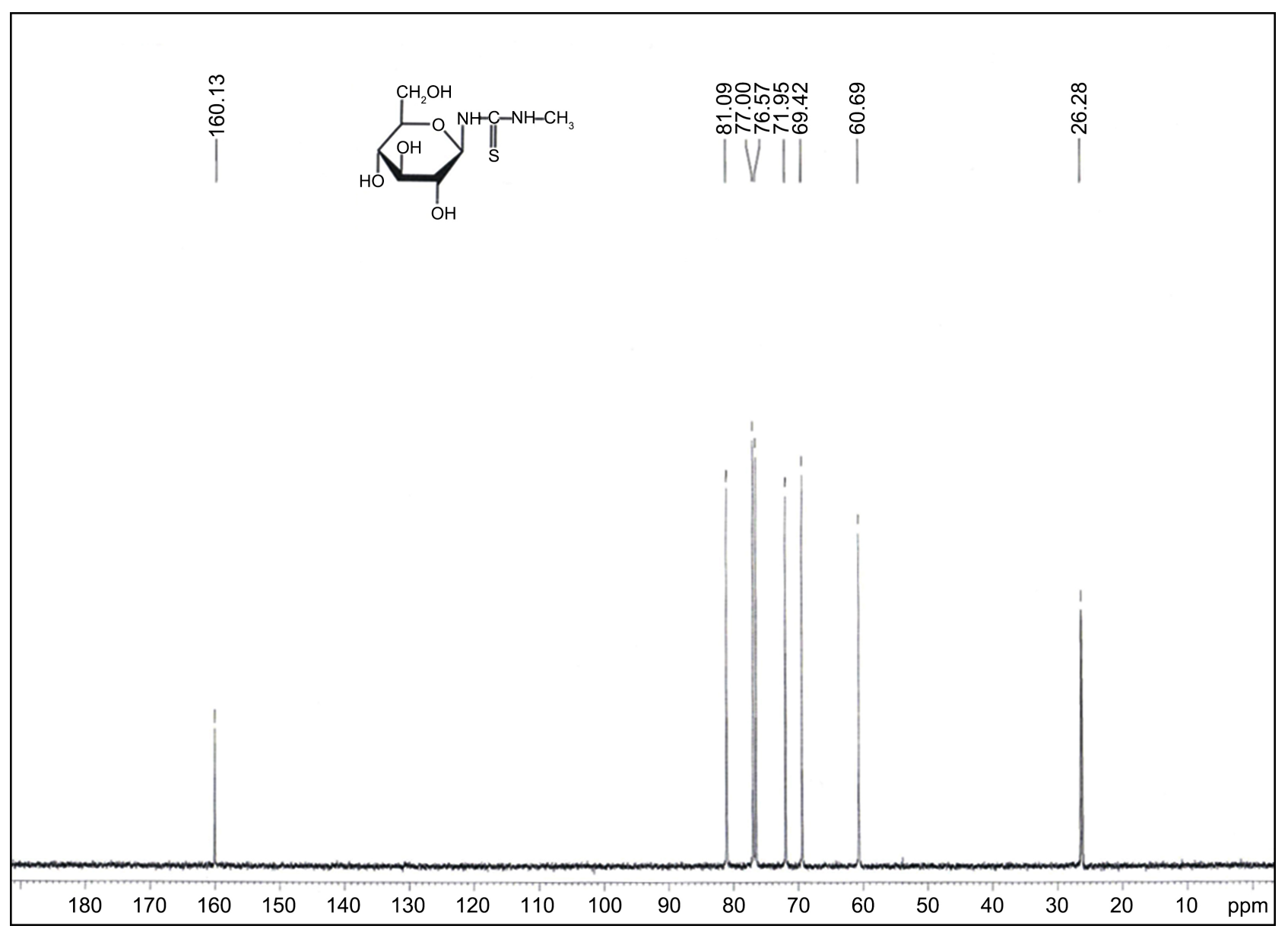

Figure 6. Pattern showing the chemical shifts for ${ }^{13} \mathrm{C}$ NMR of sugar thiocarbamides (N-methyl- $\mathrm{N}^{1}-(\beta$-D-glucopyranosyl)-thiourea). 
Table 4. Chemical shifts for ${ }^{13} \mathrm{C}$ NMR of sugar thiocarbamides.

\begin{tabular}{|c|c|c|c|c|c|c|c|c|}
\hline \multirow{2}{*}{ No. } & \multicolumn{6}{|c|}{ Carbonaceous part } & \multicolumn{2}{|c|}{ Aglycone part } \\
\hline & $\mathrm{C}_{1}$ & $\mathrm{C}_{2}$ & $\mathrm{C}_{3}$ & $\mathrm{C}_{4}$ & $\mathrm{C}_{5}$ & $\mathrm{C}_{6}$ & $\mathrm{C}=\mathrm{S}$ & Other signals \\
\hline 20 & 81.80 & 71.82 & 76.63 & 69.13 & 66.30 & - & 160.04 & $26.22(\mathrm{CH} 3)$ \\
\hline 21 & 81.09 & 71.95 & 76.57 & 69.42 & 77.00 & 60.69 & 160.13 & $26.28\left(\mathrm{CH}_{3}\right)$ \\
\hline 22 & 81.51 & 69.54 & 73.43 & 68.69 & 76.08 & 60.99 & 160.23 & $26.22(\mathrm{CH} 3)$ \\
\hline 23 & 90.5 & 73.9 & 77.0 & 67.8 & 70.1 & - & 186.1 & $\begin{array}{c}\text { 113.2; 129.2; } \\
\text { 119.2; 151.0; } \\
129.1(\mathrm{CH} \text {-arom })\end{array}$ \\
\hline 24 & 97.7 & 93.5 & 76.4 & 69.6 & 70.0 & 62.4 & 186.5 & $\begin{array}{c}113.2 ; 118.2 ; \\
119.2 ; 129.3 ; \\
128(\mathrm{CH} \text {-arom })\end{array}$ \\
\hline 25 & 88.3 & 79.1 & 74.8 & 71.2 & 74.2 & 62.0 & 186.0 & $\begin{array}{c}\text { 113.2; 118.2; } \\
\text { 119.2; 129.2; } \\
129(\mathrm{CH}-\text {-arom })\end{array}$ \\
\hline 26 & 90.9 & 77.0 & 73.9 & 70.1 & 67.8 & - & 173.7 & $\begin{array}{c}143.2 ; 144.32(\mathrm{C}-\mathrm{C}) \\
105.0(\mathrm{CH}) \\
18.3 ; 11.3\left(\mathrm{CH}_{3}\right)\end{array}$ \\
\hline 27 & 88.7 & 79.0 & 74.8 & 71.0 & 74.0 & 62.2 & 173.8 & $\begin{array}{c}143.2,144.3(\mathrm{C}-\mathrm{C}) \\
105.0(\mathrm{CH}) \\
18.3,11.2\left(\mathrm{CH}_{3}\right)\end{array}$ \\
\hline 28 & 88.5 & 79.1 & 74.7 & 71.2 & 74.2 & 62.2 & 173.7 & $\begin{array}{c}143.0 ; 144.1(\mathrm{C}-\mathrm{C}) \\
105.2(\mathrm{CH}) \\
18.2 ; 11.2\left(\mathrm{CH}_{3}\right)\end{array}$ \\
\hline 29 & 90.5 & 73.9 & 77.0 & 70.1 & 67.8 & - & 183.6 & - \\
\hline 30 & 88.3 & 74.2 & 74.8 & 71.2 & 79.1 & 62.0 & 183.5 & - \\
\hline 31 & 88.3 & 74.0 & 74.5 & 71.0 & 78.8 & 61.3 & 183.4 & - \\
\hline 32 & 87.8 & $\begin{array}{l}32.9 ; 59.4 ; \\
170.3 ; 20.7 \\
\quad(4 \mathrm{C})\end{array}$ & $\begin{array}{l}19.9 ; 65.2 ; \\
170.8 ; 20.5 \\
\quad(4 \mathrm{C})\end{array}$ & $\begin{array}{l}35.5 ; 61.4 ; \\
170.2 ; 20.8 \\
\quad(4 \mathrm{C})\end{array}$ & $\begin{array}{c}20.7 ; 66.3 \\
170.0 ; 20.3 \\
\quad(4 C)\end{array}$ & - & 183.7 & $\begin{array}{l}56.9 ; 46.0 ; \\
56.9 ; 46.2 \\
\text { (C-amine) }\end{array}$ \\
\hline
\end{tabular}

\section{Conflicts of Interest}

The authors declare no conflicts of interest regarding the publication of this paper.

\section{References}

[1] Reddy, N.S., Rao, A.S., Chari, M.A., Kumar, V.R., Jyothi, V. and Himabindu, V. (2011) Synthesis and Antibacterial Activity of Urea and Thiourea Derivatives at C-8 Alkyl Chain of Anacardic Acid Mixture Isolated from a Natural Product Cashew Nut Shell Liquid (CNSL). International Journal of Organic Chemistry, 1, 167-175. https://doi.org/10.4236/ijoc.2011.14025 
[2] Halim, A.N.A. and Ngaini, Z. (2016) Synthesis and Bacteriostatic Activities of Bis(thiourea) Derivatives with Variable Chain Length. Journal of Chemistry, Article ID: 2739832, 7 p. https://doi.org/10.1155/2016/2739832

[3] Shakeel, A., Altaf, A.A., Qureshi, A.M. and Badshah, A. (2016) Thiourea Derivatives in Drug Design and Medicinal Chemistry: A Short Review. Journal of Drug Design and Medicinal Chemistry, 2, 10-20. https://doi.org/10.11648/j.jddmc.20160201.12

[4] Mertschenk, B., Knott, A. and Bauer, W. (2013) Thiourea and Thiourea Derivatives. Ullmann's Encyclopedia of Industrial Chemistry. https://doi.org/10.1002/14356007.a26_803.pub3

[5] Shetty, P. (2017) Corrosion Inhibition Behavior of Thiourea Derivatives in Acid Media against Mild Steel Deterioration: An Overview. Surface Engineering and Applied Electrochemistry, 53, 587. https://doi.org/10.3103/S1068375517060126

[6] Shukurlaev, K.S., Baltabayev, U.A., Makhsumov, A.G. and Babaev, I.D. (2004) Anti-Inflammatory Activity of New Thiourea Derivatives. Medical Journal of Uzbekistan, 3, 154-156.

[7] Fischer, E. and Helferich, B. (1914) Synthetische Glucoside der Purine. Berichte der Deutschen Chemischen Gesellschaft, 47, 210-235.

https://doi.org/10.1002/cber.19140470133

[8] Bakirova, A.A., Dermugin, V.S., Dzhamanbaev. Z.A. and Ernazarova, B.K. (2007) Synthesis and Physicochemical Properties of Lawessons (LR) Reagent. Vestnik Kyrg. Nats.Univ. named after Zh. Balasagyn, 5, 221-224.

[9] Lecher, H.Z., Greenwood, R.A., Whitehouse, K.C. and Chao, T.H. (1956) The Phosphonation of Aromatic Compounds with Phosphorus Pentasulfide. Journal of the American Chemical Society, 78, 5018-5022. https://doi.org/10.1021/ja01600a058

[10] Dzhamanbaev, J.A., Dermugin, V.S., Bakirova, A.A. and Ernazarova, B.K. (2009) Method for Producing Glycosyl Methyl Thiourea. Kyrgyzpatent No. 1251.

[11] Dzhamanbaev, Zh.A., Dzhamanbaeva, Z.A. and Afanasyev, V.A. (1995) 4-Glycopyranosyl Semicarbazides as Intermediates for the Synthesis of Compounds, Possessing Anti-Inflammatory and Antimicrobial Activity. Rospatent No.2027721.

[12] Ozturk, T., Ertas, E. and Mert, O. (2007) Use of Lawesson's Reagent in Organic Syntheses. Chemical Reviews, 107, 5210-5278. https://doi.org/10.1021/cr040650b

[13] Popović-Djordjević, J.B., Jevtić, I.I., Grozdanić, N.D., Šegan, S.B., Zlatović, M.V., Ivanović, M.D. and Stanojković, T.P. (2017) $\alpha$-Glucosidase Inhibitory Activity and Cytotoxic Effects of Some Cyclic Urea and Carbamate Derivatives. Journal of Enzyme Inhibition and Medicinal Chemistry, 32, 298-303. https://doi.org/10.1080/14756366.2016.1250754

[14] Price, N.P.J. (2004) Acylic Sugar Derivatives for GC/MS Analysis of ${ }^{13}$ C-Enrichment during Carbohydrate Metabolism, Analytical Chemistry, 76, 6566-6574. https://doi.org/10.1021/ac049198m 